\title{
Long-range Stress Redistribution Resulting from Damage in Heterogeneous Media
}

\author{
Yilong Bai $^{1}$, ZhaOke JiA ${ }^{1}$, XiaOhui Zhang ${ }^{1}$, Fujiu Ke ${ }^{1,2}$, \\ and MENGFEN XIA ${ }^{1,3}$
}

\begin{abstract}
It has been shown in CA simulations and data analysis of earthquakes that declustered or characteristic large earthquakes may occur with long-range stress redistribution. In order to understand long-range stress redistribution, we propose a linear-elastic but heterogeneous-brittle model. The stress redistribution in the heterogeneous-brittle medium implies a longer-range interaction than that in an elastic medium. Therefore, it is surmised that the longer-range stress redistribution resulting from damage in heterogeneous media may be a plausible mechanism governing main shocks.
\end{abstract}

Key words: Long-range stress redistribution, damage, heterogeneous media.

\section{Introduction}

Recently, the significance of long-range stress redistribution in understanding the earthquake mechanism has drawn considerable attention (HiLl et al., 1993; KLEIN et al., 2000; WeATHERLeY et al., 2000; RundLE, J.B. 1995; KNOPOFF, 2000). Various models of cellular automata (CA) with long-range stress redistribution for earthquake faults were widely used in these studies. Nonetheless, determination of the nature and significance of the long-range interaction is by no means an easy problem. Different research groups have different understandings. For instance, KLEIN et al. (2000) stated that "linear elasticity yields long-range stress tensors for a variety of geological applications" and "for a two-dimensional dislocation in a threedimensional homogeneous elastic medium, the magnitude of the stress tensor goes as $\sim 1 / \mathrm{r}^{3}$." They noticed "while geophysicists do not know the actual stress tensors for real faults, they expect that long-range stress tensors, which are similar to the $\sim 1 / \mathrm{r}^{3}$ interaction, apply to faults." Moreover, they stressed that "it is suspected that microcracks in a fault, as well as other "defects" such as water, screen the

\footnotetext{
${ }^{1}$ State Key Laboratory of Nonlinear Mechanics, Institute of Mechanics, Chinese Academy of Sciences, Beijing 100080, China. E-mail: baiyl@Inm.imech.ac.cn

${ }^{2}$ Department of Applied Physics, Beijing University of Aeronautics and Astronautics, Beijing 100083, China.E-mail: kefj@Inm.imech.ac.cn

${ }^{3}$ Department of Physics, Peking University, Beijing 100871, China. E-mail: xiam@1nm.imech.ac.cn
} 
$\sim 1 / \mathrm{r}^{3}$ interaction, leading to a proposed $\sim e^{-\alpha \mathrm{r}} / r^{3}$ interaction, where $\alpha<<1$, implying a slow decay to the long-range interaction over the fault's extent."

On the contrary, WeAtherley et al. (2000) pointed out in their cellular automaton model that "the interaction exponent $\left(p\right.$ in $\sim 1 / r^{p}$ ) determines the effective range for strain redistribution in the model. The effective range decreases rapidly as the exponent $(p)$ increases. The event-size distributions illustrate three different populations of events in the dissipative healing models (two-dimensional models):

- Characteristic large events $(p<1.5)$,

- Power-law scaling events $(1.5<p \leq 2.0)$,

- Overdamped, no large events $(p>2.0)$.

They concluded that the models display a smooth transition from characteristic large events preceded by strain correlation evolution and accelerating energy release, to a power-law distribution of events preceded by linear energy release, as the effective range of interactions decreases. Given that the stress redistributions in three and two-dimensional homogeneous linear elastic media are $\sim 1 / r^{3}$ and $\sim 1 / r^{2}$ respectively, the difficulty in understanding long-range stress redistribution is obvious.

Physically, the existence of cracks and other "defects" like water may have two opposite effects on stress redistribution. One is to screen stress and then lead to a shorter-range redistribution, as claimed by KLEIN et al. (2000). On the other hand, the stress balance requires a compensational increase of the stress beyond the "defects," implying a longer-range redistribution of stress.

Recently, KNOPOFF (2000) investigated the magnitude distribution of declustered earthquakes in Southern California. He concluded that the characteristic length of $3 \mathrm{~km}$ in the magnitude distribution is a crossover between two different mechanisms in the physics of earthquake occurrence.

All of the results remind us that a declustered or characteristic large earthquake may occur relevant to some intrinsic length scales and with a longer-range stress redistribution. Then, instead of the commonly used homogeneous linear elastic theory, can we find possible alternative models with longer-range stress redistribution? This is the aim of this paper.

\section{Possible Long-range Stress Redistribution in Heterogeneous Media}

It is well known that the main terms of stress in a homogeneous linear elastic medium are $\sim 1 / r^{3}$ in a three-dimension model with a spherical void and $\sim 1 / r^{2}$ in a twodimensional model with a cylindrical hole. Now, let us examine the stress redistribution owing to a void in a linear-elastic but heterogeneous-brittle medium to determine the effect of microdamage resulting from the heterogeneity on stress redistribution. In particular, we wish to determine whether microdamage is a 
"screen" leading to a shorter-range redistribution or a compensation implying a longer-range redistribution of stress.

It is assumed in the model that every mesoscopic element has the same elastic moduli, like Young's modulus $E$ and Poisson ratio $v$, but various breaking strengths $\sigma_{c}$ or strain threshold $\varepsilon_{c}$. Moreover, the strain threshold of the element follows a distribution function (Fig. 1),

$$
h\left(\varepsilon_{c}\right)=\left\{\begin{array}{lll}
0 ; & \text { when } \quad \varepsilon_{c}<\varepsilon_{c}^{*} \\
\frac{q-1}{\varepsilon_{c}^{*}}\left(\frac{\varepsilon_{c}}{\varepsilon_{c}^{*}}\right)^{-q}, q>1 ; & \text { when } \quad \varepsilon_{c} \geq \varepsilon_{c}^{*}
\end{array}\right.
$$

where $\varepsilon_{c}^{*}$ is the minimum of strain threshold, i.e., the strain thresholds of all elements are larger than $\varepsilon_{c}^{*}$, and the number of elements with strain threshold higher than $\varepsilon_{c}^{*}$ decreases as a power law. Then, for strain less than $\varepsilon_{c}^{*}$, all mesoscopic elements remain solid, namely the system is elastic; however, as the strain is higher than $\varepsilon_{c}^{*}$, some mesoscopic elements will break and damage occurs. Also, the parameter $q$ in the heterogeneous-brittle model should remain greater than unit and the greater the parameter $q$ is, the stronger the heterogeneity relevant damage is, see Figure 1.

We must confess that we do not know the actual distribution of strength in geological media. However, the above simple distribution looks qualitatively reasonable and makes it easy to perform some analysis to study stress redistribution in heterogeneous media. In fact, the heterogeneity must imply some intrinsic length scales relevant to structures of geological media. Nonetheless, as first-order approximation, we use local mean field to deal with the problem. In this way, the intrinsic length scales are eliminated in the approximation and the stress-strain relation in uniaxial stress state is (Fig. 2),

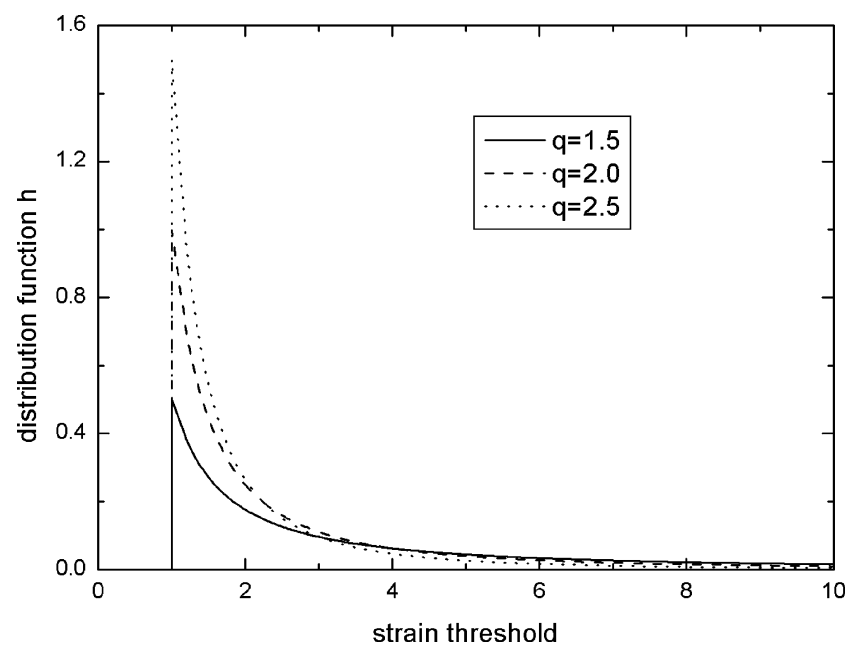

Figure 1

The distribution function of strength of mesoscopically heterogeneous elements, $h\left(\varepsilon_{c}\right)$. It shows that the greater the value of $q$ is, the smaller the mesoscopic strength scatter becomes. 


$$
\begin{gathered}
\sigma= \begin{cases}E \varepsilon ; & \text { when } \quad \varepsilon_{c}<\varepsilon_{c}^{*} \\
E \varepsilon_{c}^{*}\left(\frac{\varepsilon}{\varepsilon_{c}^{*}}\right)^{2-q} & \text { when } \quad \varepsilon_{c} \geq \varepsilon_{c}^{*}\end{cases} \\
D=\int_{0}^{\varepsilon} h\left(\varepsilon_{c}\right) \mathrm{d} \varepsilon_{c}=\left\{\begin{array}{lll}
0 ; & \text { when } & \varepsilon_{c}<\varepsilon_{c}^{*} \\
1-\left(\frac{\varepsilon}{\varepsilon_{c}^{*}}\right)^{1-q} ; & \text { when } & \varepsilon_{c} \geq \varepsilon_{c}^{*}
\end{array}\right.
\end{gathered}
$$

where $D$ is damage. In accord with damage mechanics, the effect of damage can be described by the reduced modulus, such as

$$
E^{\prime}=E(1-D)=E \bar{\varepsilon}^{-1-q}
$$

where $\bar{\varepsilon}=\left(\frac{\varepsilon}{\varepsilon_{c}^{*}}\right)$.

In multi-axial stress state, it is presumed that the damage or the reduced moduli are governed by maximum strain, i.e., the circumferential strain $\varepsilon_{\theta}$. We call this the $\theta$-model. Then, when $\bar{\varepsilon}_{\theta}>1$, the elastic-brittle constitutive relation in spherical configuration (3-D) becomes

$$
\begin{gathered}
\bar{\sigma}_{r}=\left[(1-v) \bar{\varepsilon}_{r}+2 v \bar{\varepsilon}_{\theta}\right] \bar{\varepsilon}_{\theta}^{1-q} \\
\bar{\sigma}_{\theta}=\left[v \bar{\varepsilon}_{r}+\bar{\varepsilon}_{\theta}\right] \bar{\varepsilon}_{\theta}^{1-q}
\end{gathered}
$$

where $v$ is Poisson ratio and $\bar{\sigma}=(1-2 v)(1+v) \sigma / E \varepsilon_{c}^{*}$. In the following we will ignore the bar above all dimensionless variables. The stress balance equation in spherical configuration (3-D) is

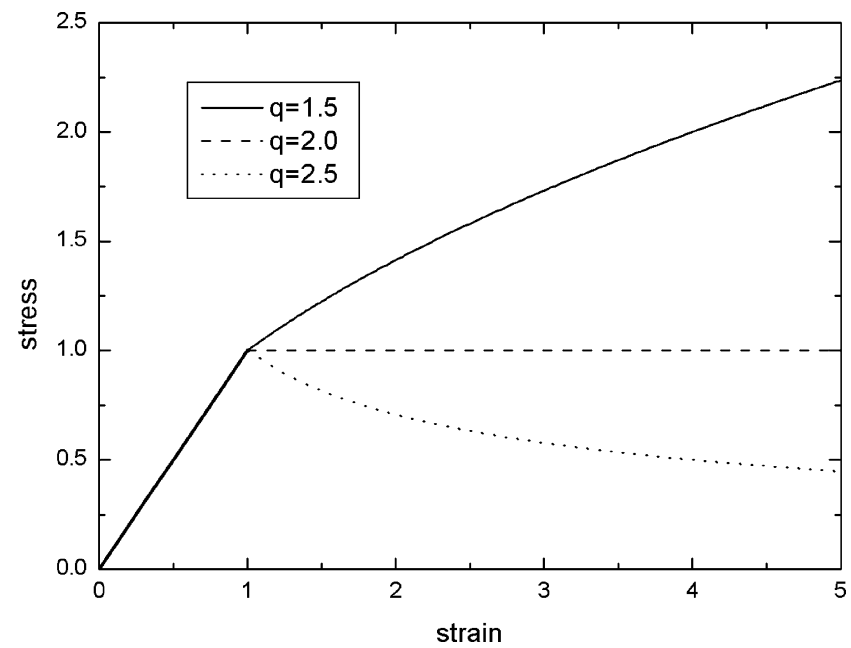

Figure 2

The one-dimensional stress and strain relation of the linear-elastic but heterogeneous-brittle model with different $q$ values. It shows that the greater the mesoscopic strength scatter is, the softer the model becomes. 


$$
\frac{d \sigma}{d r}+2 \frac{\sigma_{r}-\sigma_{\theta}}{r}=0
$$

The strains can be expressed by dimensionless displacement $u$ and radius $r$ (all are nondimensionalized by the inner radius of the spherical void, Figure 3),

$$
\begin{gathered}
\varepsilon_{r}=\frac{d u}{d r}, \\
\varepsilon_{\theta}=\frac{u}{r},
\end{gathered}
$$

Substitution of the strain definition (8-9) and the elastic-brittle relation (5-6) into the balance equation (7) leads to a nonlinear ordinary differential equation (Lambert equation),

$$
(1-v) u^{\prime \prime}+(1-v)(1-q) \frac{u^{\prime 2}}{u}+(1+q+v-3 v q) \frac{u^{\prime}}{r}+2(v q-1) \frac{u}{r^{2}}=0 .
$$

Equation (10) works for the presumed $\theta$-model with constitutive relation ( 5 and 6 ). It is worth noting that Eq. (10) reduces to the linear elastic version, when $q=1$. However, when $q$ is greater than unity, the nonlinear second term in Eq. (10) plays a significant role. We use the following nonlinear transformation to simplify equation (10). Let

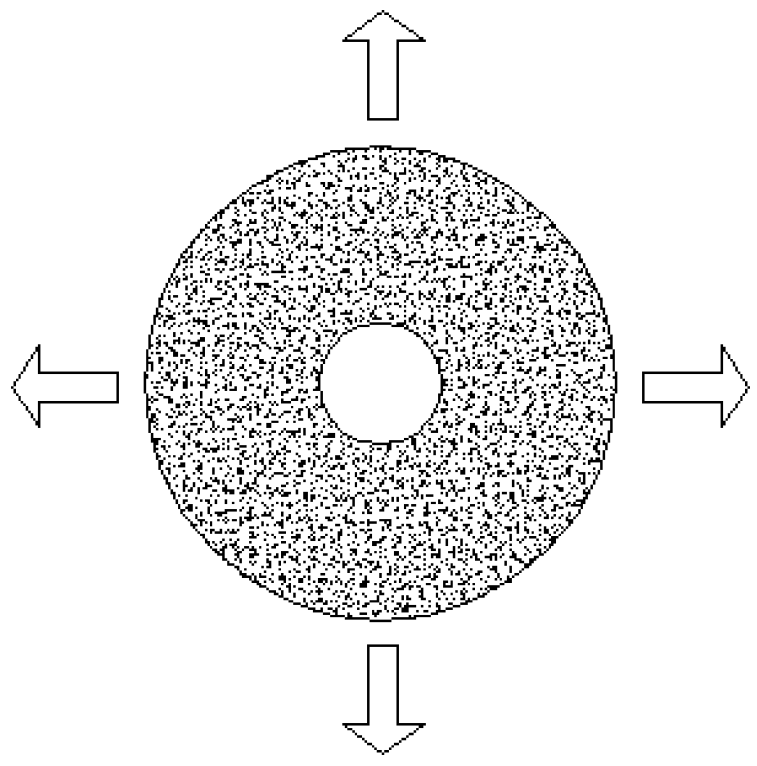

Figure 3

The configuration for the discussion of stress redistribution in heterogeneous media. The central void represents initial damage. The grayness indicates heterogeneity, and the big circle shows a two-dimensional axisymmetric configuration of the model. 


$$
u=U^{\alpha}
$$

where $\alpha$ is an undetermined parameter. After substituting (11) into Eq. (10) and taking $\alpha=1 /(2-q)$, one can find that the equation for variable $U$ becomes a linear one,

$$
(1-v) \alpha U^{\prime \prime}+(1+q+v-3 v q) \alpha \frac{U^{\prime}}{r}+2(v q-1) \frac{U}{r^{2}}=0 .
$$

There is a power-law solution to Eq. (12) of the form,

$$
U=A r^{-\beta},
$$

where $A$ is an arbitrary constant and $\beta$ is an undetermined exponent. Substitution of the solution (13) into equation (12) gives the following dependence of the exponent $\beta$ on $q$ and $v$,

$$
\beta_{1}=q-2 \text { and } \beta_{2}=\frac{2(1-v q)}{1-v} .
$$

Hence, the stresses, either circumferential or radial, will be in the form

$$
\sigma=\left[O(1)+O\left(\frac{\varepsilon_{r}}{\varepsilon_{\theta}}\right)\right] \varepsilon_{\theta}^{2-q}=\left[O(1)+O\left(\frac{\varepsilon_{r}}{\varepsilon_{\theta}}\right)\right]\left[A_{1}+A_{2} r^{-p}\right],
$$

where $A_{1}$ and $A_{2}$ are two arbitrary constants, and

$$
p=\beta+2-q=2 \frac{2-v}{1-v}-\frac{1+v}{1-v} q .
$$

One can verify that the ratio of strains in the expression of stress (15),

$$
O\left(\frac{\varepsilon_{r}}{\varepsilon_{\theta}}\right) \approx O(1)
$$

The reason is as follows. Generally speaking, the solution of the variable $U$ can be written as

$$
U=A_{1} r^{-\beta_{1}}+A_{2} r^{-\beta_{2}}
$$

Then, the term

$$
\left(\frac{\varepsilon_{r}}{\varepsilon_{\theta}}\right)=-\alpha \beta_{1} \frac{1+\frac{A_{2} \beta_{2}}{A_{1} \beta_{1}} r^{-\beta_{2}+\beta_{1}}}{1+\frac{A_{2}}{A_{1}} r^{-\beta_{2}+\beta_{1}}}
$$

Provided $\beta_{2}>\beta_{1}$,

$$
O\left(\frac{\varepsilon_{r}}{\varepsilon_{\theta}}\right) \approx O(1)+O\left(r^{-\beta_{2}+\beta_{1}}\right)
$$


So,

$$
\sigma \approx A_{1}^{\prime}+A_{2}^{\prime} r^{-2 \frac{2-v}{1-v}-\frac{1+v}{1-v} q}
$$

Notably, the power-law exponent $p$ in stress redistribution, see Eq. (16) or (21), approaches 3 when $q$ tends to 1 , as linear homogeneous elasticity gives in textbook, and $p$ decreases with increasing $q$. That is to say, stress redistribution in a heterogeneous elastic-brittle medium has longer interaction range with stronger heterogeneity relevant damage.

Similarly, we have derived the stresses for the two-dimensional (cylindrical) configuration under the same assumptions of heterogeneity and reduced modulus. The corresponding versions in the two-dimensional plane stress case (2-D) are as follows:

The elastic-brittle constitutive relation when $\bar{\varepsilon}_{\theta}>1$ becomes

$$
\begin{aligned}
& \bar{\sigma}_{r}=\left[\bar{\varepsilon}_{r}+v \bar{\varepsilon}_{\theta}\right] \bar{\varepsilon}_{\theta}^{1-q}, \\
& \bar{\sigma}_{\theta}=\left[v \bar{\varepsilon}_{r}+\bar{\varepsilon}_{\theta}\right] \bar{\varepsilon}_{\theta}^{1-q},
\end{aligned}
$$

where $\bar{\sigma}=\left(1-v^{2}\right) \sigma / E \varepsilon_{c}^{*}$. Later we ignore the bar above dimensionless variables again. The stress balance equation in cylindrical configuration (2-D) is

$$
\frac{d \sigma}{d r}+\frac{\sigma_{r}-\sigma_{\theta}}{r}=0
$$

The non-linear ordinary differential equation of displacement $u$ is.

$$
u^{\prime \prime}+(1-q) \frac{u \prime^{2}}{u}+(q+v-v q) \frac{u^{\prime}}{r}+(v q-1-v) \frac{u}{r^{2}}=0 .
$$

The corresponding power-law exponent $p$ is

$$
p=\beta+2-q=\left\{\begin{array}{l}
0 \\
3+v-(1+v) q
\end{array} .\right.
$$

Similarly, the power-law exponent $p$ in stress redistribution, see Eq. (22), approaches 2 when $q$ tends to 1 and $p$ decreases with increasing $q$ also, although at a slower rate than that in three-dimensions (Table 1).

\section{Results, Finite Element Computation and Discussions}

Before discussing concrete calculated results, certain remarks on the stress redistribution in heterogeneous media obtained in the previous section should be emphasized.

Noticeably, the obtained power distribution of stresses in the model should be testified to be at least a proper approximation of real geological media. However, 
Table 1

The formula and values of power exponent $p$ in approximate power law, $\sigma \approx r^{-p}$ in three-and two-dimensional configurations

\begin{tabular}{ccc}
\hline$q$ & $\begin{array}{c}p(3-\mathrm{D}) \\
p=2 \frac{2-v}{1-n u}-\frac{1+v}{1-v} q\end{array}$ & $\begin{array}{c}p(2-\mathrm{D}) \\
\end{array}$ \\
& 3 & when $v=1 / 4$ \\
\hline 1 & 2.66 & 2 \\
1.2 & 2.16 & 1.75 \\
1.5 & 1.75 & 1.37 \\
1.75 & & 1.06 \\
\hline
\end{tabular}

geophysicists do not know the actual stress tensors for real faults. Therefore we have to consider all possible models and resort to numerical simulations. The two major assumptions made in the $\theta$ - model are: Poisson ratio $v$ remains invariant and the circumferential strain $\varepsilon_{\theta}$ governs the reduced moduli. In order to check the significance of the second assumption in the $\theta$-model, we also calculate an alternative model - the mixed model, termed the M-model, which consists of elastic and damaged deformations. In the three-dimensional configuration,

$$
\begin{gathered}
\sigma_{r}=\left[(1-v) \varepsilon_{r}+2 v \varepsilon_{\theta}\right] \\
\sigma_{\theta}=\left[v \varepsilon_{\theta}+\varepsilon_{\theta}\right] \epsilon_{\theta}^{1-q},
\end{gathered}
$$

and in the two-dimensional case,

$$
\begin{gathered}
\sigma_{r}=\left[\varepsilon_{r}+v \varepsilon_{\theta}\right] \\
\sigma_{\theta}=\left[v \varepsilon_{r}+\varepsilon_{\theta}\right] \varepsilon_{\theta}^{1-q} .
\end{gathered}
$$

Figures 4 and 5 show the comparisons of circumferential (Fig 4) and radial (Fig 5) stresses with radial distance for $q=1.2$ in the $\theta$-model, mixed model and the elastic one, respectively. It is clear that the two damage models present slower attenuation than that of the elastic one. As another comparison, Figure 6 gives circumferential stress with radial distance for $q=1.5$, in the three models, respectively. One can notice that with increasing $q$ value, i.e., in the more damaged medium, the stress demonstrates even slower attenuation

In addition, finite element numerical simulations in two dimension were made. The simulation is implemented by ABAQUS (a nonlinear Finite Element Analysis package). We use an 8-node reduced-integration axisymmetric element to solve the problem. The damage constitutive relation is based on the $\theta$-model. In this way we cannot only check the calculations based on the obtained approximate analytic solutions but also examine the transition from elastic to damage models with increasing loading, see Figure 7.

The comparison of all these calculations shows a clear trend of longer range of stress redistribution with increasing index $q$, although the M-model and the full 


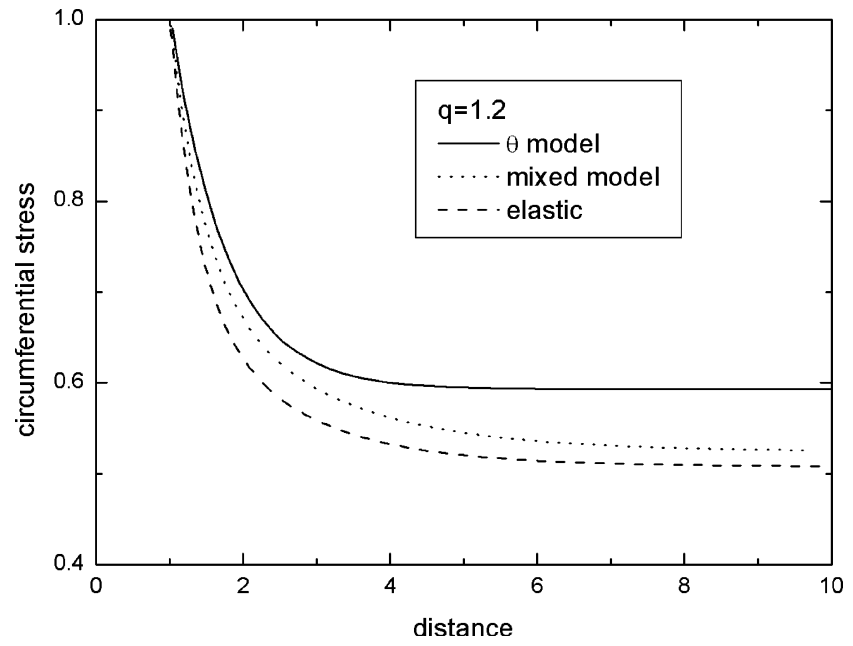

Figure 4

The variations of circumferential stresses with radial distance for $q=1.2$, in $\theta$-model (solid), mixed model (dotted) and elastic (dashed), respectively. For easy comparison, all stresses are renormalized by the stress value at the inner surface of the hole.

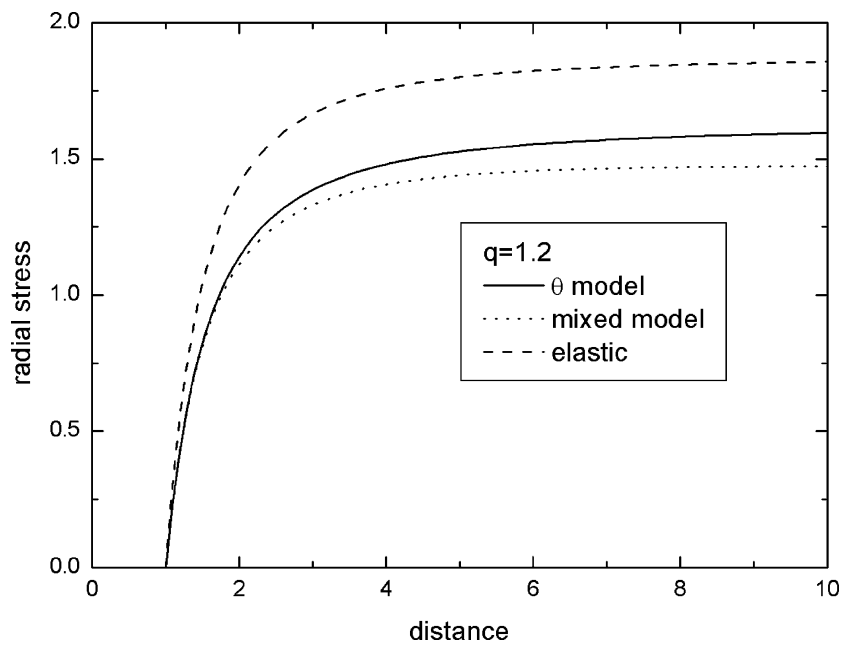

Figure 5

The variations of radial stresses with radial distance for $q=1.2$, in $\theta$-model (solid), mixed model (dotted) and elastic (dashed), respectively.

numerical simulations show considerably more complicated behavior than the simple power law of stress redistribution in the $\theta$-model. Based on these results, the stress redistribution in heterogeneous media with interaction range longer than in linear 


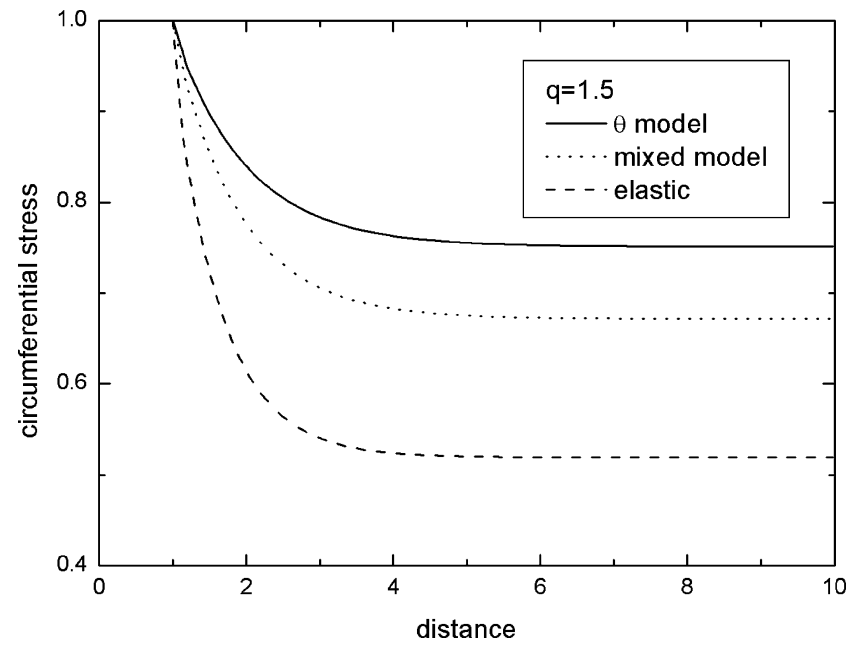

Figure 6

The variations of circumferential stresses with radial distance for $q=1.5$, in $\theta$-model (solid), mixed model (dotted) and elastic (dashed), respectively. For easy comparison, all stresses are renormalized by the stress value at the inner surface of the hole.

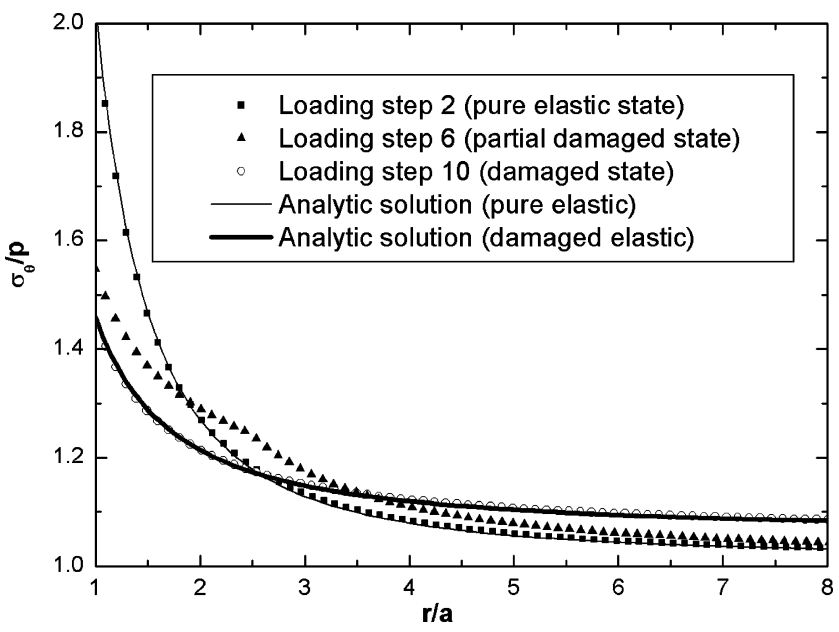

Figure 7

The variations of circumferential stress with radial distance for the elastic-heterogeneous brittle medium with parameters $q=1.5$ and $v=0.25$ for various loading steps. The points are the FE results. The two solid lines are the analytical results of elastic and the damaged elastic-brittle models, respectively. The agreements between FE and analytic solutions are very good. The dotted line in between is the FE result for the state of partly elastic (outer part) and partly damaged (inner part).

homogeneous elastic medium (like $p=3$ in three dimension) might be a reasonable mechanism rather than a virtual assumption. In addition, we could apply these longrange interactions to cellular automata to simulate earthquakes. 
Then, what is the physical basis of the obtained results? As mentioned in the introduction, long-range stress redistribution may imply some intrinsic length scales. Physically, intrinsic length scales in heterogeneous media may be cataloged into two groups: small ones $\delta$ of mesoscopic heterogeneities or microcracks, and large ones $l$ of macroscopic faults, which follow $\delta \ll \Delta \ll l$, where $\Delta$ is the length scale of representative element volume (REV) in the calculation model and tends to become infinitesimally small in the continuum approximation. All intrinsic structures with length scales $\delta$ are averaged into the distribution function of heterogeneity and become hidden in the present mean field approximation of the model. Their effect is to bring about the stress redistribution with longer range, owing to less load-supporting ability in the damaged REV. This is what we modeled in the present paper. However, large macroscopic faults with length scales $l \gg \Delta$ should be the free interface in the concerned body. Clearly, these macroscopic free internal boundaries would screen stress field. Therefore, we assume that the long-range stress redistribution resulting from damage in heterogeneous media with intrinsic length scales to quite possibly be a mechanism governing main shocks.

\section{Concluding Remarks}

In order to understand why a declustered or characteristic large earthquake may occur relevant to some intrinsic length scales observed in earthquake data and with a longer-range stress redistribution observed in CA simulations, we propose a linearelastic but heterogeneous-brittle model. The stress redistribution owing to damage in the heterogeneous-brittle medium has a power-law exponent in the $\theta$-model, of $p=2 \frac{2-v}{1-v}-\frac{1-v}{1-v} q$ in three dimensions and $p=3+v-(1+v) q$ in two dimensions, respectively instead of 3 and 2 in elastic medium, hence implying longer-range interactions. Other calculations, like finite element simulations, also show a clear trend of longer range of stress redistribution with increasing index $q$, although more complicated than simple power law. Therefore, it is thought that the long-range stress redistribution resulting from damage in heterogeneous media may quite possibly be a mechanism governing main shocks.

\section{Acknowledgement}

This work is granted by the National Natural Science Foundation of China (NSFC-10172084 and NSFC-10232050) and Major State Research Project G200007735. 


\section{REFERENCES}

BAI, Y. L., XIA, M. F., KE, F. J., and LI, H. L. (2002), Non-equilibrium evolution of collective microdamage and its coupling with mesoscopic heterogeneities and stress fluctuations. In Shock Dynamics and Nonequilibrium Mesoscopic Fluctuations in Solids (eds. Horie, Y. Thadhani, N. and Davison, L., SpringerVerlag (to appear).

BAI, Y. L., XIA, M. F., Ke, F. J., and Li, H. L. (2002), Closed Trans-scale Statistical Microdamage Mechanics, Acta Mechanica Sinica 18, 1-17.

Hill, D. P., Reasenberg, P. A., Michael, A., Arabaz, W. J., Beroza, G. and et al. (1993), Seismicity Remotely Triggered by the Magnitude 7.3 Landers, California, Earthquake, Science 260, 1617-1623.

Klein, W., Anghel, M., Ferguson, C. D., Rundle, J. B., and Sa Martins, J. S. (2000), Statistical analysis of a model for earthquake faults with long-range stress transfer. In Geocomplexity and the Physics of Earthquakes (eds Rundle J.B., Turcotte, D. and Klein, W.,) AGU 2000.

KNOPOFF, L. (2000), The Magnitude Distribution of Declustered Earthquakes in Southern California, PNAS 97, 11,880-11,884.

Rundle, J. B. and Klein, W. (1995), Dynamical segmentation and rupture patterns in a "toy" slider block model for earthquakes, Nonlinear Proc. In GeoPhysics, 2, 61-81.

Weatherley, D., Xia, M. F., and Mora, P. (2000), Dynamical Complexity in Cellular Automata with Long-range Stress Transfer, AGU 2000.

(Received September 27, 2002, revised February 28, 2003, accepted March 7, 2003)

To access this journal online:
http://www.birkhauser.ch

